\title{
EARLY MORPHOLOGICAL DIVERSITY IN BLASTOZOAN ECHINODERMS
}

FOOTE* ${ }^{\star}$ Mike, Museum of Paleontology, University of Michigan, Ann Arbor, MI 48109 U.S.A.

Morphological diversity (MD) is important for understanding a clade's evolution and for comparing clades to discover evolutionary laws. As the scale of analysis (taxonomic rank) increases, defining a morphological space becomes less tractable, and assessing MD becomes more difficult. Continuous morphometric variables are hard to define for very disparate species, but morphological dissimilarity can be assessed by discrete characters. I use this approach to document the history of MD in the echinoderm subphlylum Blastozoa.

About 60 discrete characters were defined, relating to: arrangement and number of thecal plates; shape and symmetry of theca; number, arrangement, form, and plating of ambulacra; arrangement, form, and plating of brachioles; form and plating of attachment structure; nature of respiratory structures; and position of mouth and anus. Character difference for binary and unordered multistate characters is 0 for matches and 1 for mismatches. Ordered multistate characters were scaled so that the maximum character difference was unity. Pairwise dissimilarity between species was measured by the mean character difference. MD in a stratigraphic interval was measured by the mean pairwise dissimilarity among species. Over half of all described genera were represented. MD involves not only the origin of new character states but also the maintenance of primitive states in a clade; therefore, assessment of MD was deliberately not restricted to analysis of novelties! The figures below compare MD to generic diversity based on the Treatise and post-Treatise monographs. Debate persists about the nature and extent of early echinoderm diversification. Most pertinent to this debate, MD in the Cambrian and Lower Ordovician is extremely high compared to taxonomic diversity; there are few taxa sparsely occupying much morphospace. Analysis of clade-shape statistics indicates that the blastozoan clade is bottom-heavy in both aspects of diversity, but more so with respect to $M D$. The results overall are not sensitive to the details of the time scale, the inclusion of problematic forms, or the number of characters used. The general picture agrees with that advocated by sprinkle (among others) for the echinoderms as a whole.
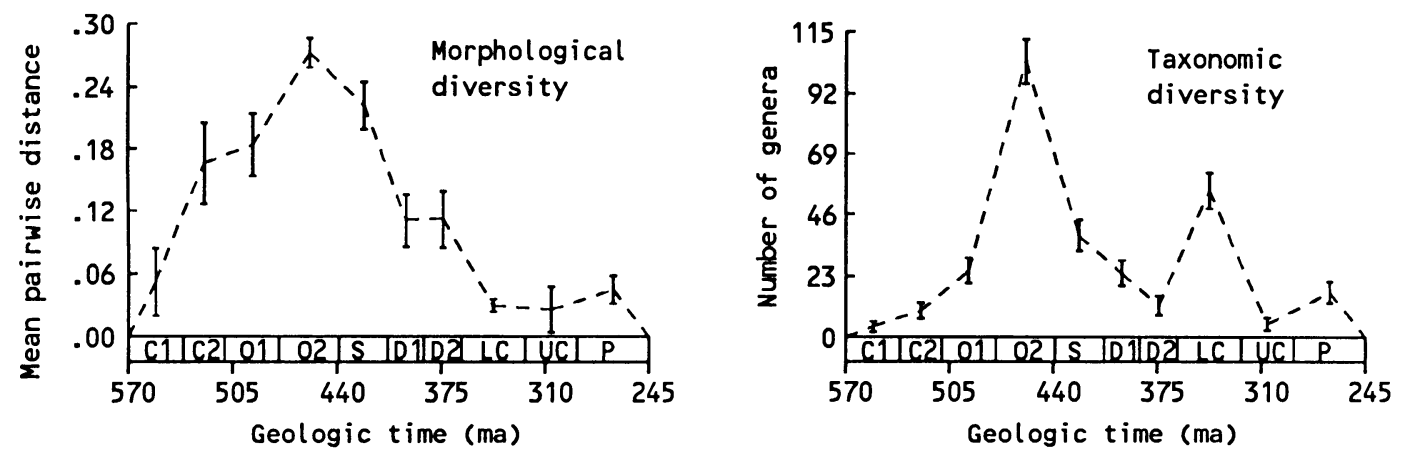